\title{
Arthroscopic Treatment of Septic Arthritis of the Shoulder: Technical Pearls to Reduce the Rate of Reoperation
}

\author{
Ji Eun Kwon ${ }^{1}$, Ji Soon Park ${ }^{2}$, Hae Bong Park ${ }^{3}$, Kyung Pyo Nam ${ }^{4}$, Hyuk Jun Seo ${ }^{5}$, Woo Kim ${ }^{6}$, Ye Hyun Lee ${ }^{1}$, \\ Young Dae Jeon", Joo Han $\mathrm{Oh}^{7}$ \\ ${ }^{1}$ Department of Orthopedic Surgery, National Police Hospital, Seoul, Korea \\ ${ }^{2}$ Department of Orthopedic Surgery, Healthpoint Hospital, Abu Dhabi, UAE \\ ${ }^{3}$ Department of Orthopedic Surgery, Human Bone Orthopedic Clinic, Incheon, Korea \\ ${ }^{4}$ Department of Orthopedic Surgery, Yeson Hospital, Bucheon, Korea \\ ${ }^{5}$ Department of Orthopedic Surgery, Daegu Chamtntn Hospital, Daegu, Korea \\ ${ }^{6}$ Department of Orthopedic Surgery, Seoulkiwoonchan Orthopedic Clinic, Seoul, Korea \\ ${ }^{7}$ Department of Orthopedic Surgery, Seoul National University Bundang Hospital, Seoul National University College of Medicine, Seongnam, Korea
}

Background: The aim of this study was to evaluate clinical experience with arthroscopic debridement for septic arthritis of the shoulder joint and to report on our patient outcomes.

Methods: The retrospective analysis included 36 shoulders (male:female, 15:21), contributed by 35 patients (mean age, 63.8 years) treated by arthroscopy for septic arthritis of the shoulder between November 2003 and February 2016. The mean follow-up period was 14.3 months (range, 12-33 months). An additional posterolateral portal and a $70^{\circ}$ arthroscope was used to access the posteroinferior glenohumeral (GH) joint and posteroinferior subacromial (SA) space, respectively. Irrigation was performed with a large volume of fluid (25.1 \pm 8.1 L). Multiple suction drains (average, 3.3 drains) were inserted into the GH joint and SA space and removed $8.9 \pm 4.3$ days after surgery. Intravenous antibiotics were administered for $3.9 \pm 1.8$ weeks after surgery, followed by oral antibiotic treatment for another $3.6 \pm 1.9$ weeks.

Results: Among the 36 shoulders, reoperation was required in two cases (5.6\%). The average range of motion achieved was $150.0^{\circ}$ for forward flexion and T9 for internal rotation. The mean simple shoulder test score was $7.9 \pm 3.6$ points. Nineteen shoulders (52.8\%) had acupuncture or injection history prior to the infection. Pathogens were identified in 15 shoulders, with Staphylococcus aureus being the most commonly identified pathogen (10/15). Both the GH joint and the SA space were involved in 21 shoulders, while 14 cases involved only the $\mathrm{GH}$ joint and one case involved only the SA space.

Conclusions: Complete debridement using an additional posterolateral portal and $70^{\circ}$ arthroscope, a large volume of irrigation with $>20 \mathrm{~L}$ of saline, and multiple suction drains may reduce the reoperation rate.

Keywords: Shoulder; Septic arthritis; $70^{\circ}$ Arthroscope; Posterolateral portal

Received: November 23, 2019 Revised: January 19, 2020 Accepted: January 24, 2020

Correspondence to: Young Dae Jeon

Department of Orthopedic Surgery, Seoul National University Bundang Hospital, 82 Gumi-ro 173beon-gil, Bundang-gu, Seongnam 13620, Korea Tel: +82-31-787-7199, Fax: +82-31-787-4095, E-mail: yd.jeon84@gmail.com, ORCID: https://orcid.org/0000-0003-4862-9679

Financial support: None.

Conflict of interest: None.

Copyright@ 2020 Korean Shoulder and Elbow Society. All Rights Reserved.

This is an Open Access article distributed under the terms of the Creative Commons Attribution Non-Commercial License (http://creativecommons.org/licenses/by-nc/4.0/) which permits unrestricted non-commercial use, distribution, and reproduction in any medium, provided the original work is properly cited. 


\section{INTRODUCTION}

Septic arthritis of the shoulder joint is a rare condition, accounting for up to $3 \%$ of all joint infections [1]. However, the importance should not be understated due to its association with specific health comorbidities (e.g., diabetes, liver cirrhosis, and malignancy), the high rate of mortality if not treated promptly, the potential for long-term morbidities (including osteomyelitis, arthritis, and shoulder stiffness), and increased incidence following needle placement around the shoulder joint [1-8]. Treatment options for septic arthritis of the shoulder joint include repetitive aspiration, open arthrotomy with debridement, and arthroscopic debridement and irrigation [1-4,7]. Of these, arthroscopic debridement and irrigation has become popular owing to its many advantages, including the use of a small incision, lower level of postoperative pain, better visualization of the joint compared to an open surgical approach, and overall good clinical results [3,5,9-11]. However, the rate of reoperation after arthroscopic management remains high at $26 \%$ to $32 \%[3,11]$. A recent article discussed the risk factors for failure of a single surgical debridement. All factors identified were preoperative in nature and were therefore not controllable by the treatment strategy or the operative method, and none of the factors identified could effectively enhance patient prognosis [12]. Therefore, our aim in this study was to describe our clinical experience with arthroscopic debridement for septic arthritis of the shoulder joint and to report on our patient outcomes. Based on our experience, we describe our novel surgical protocol for reducing the rate of reoperation and include a comparison of our outcomes to previously published data.

\section{METHODS}

This study, a retrospective review, was approved by the Institutional Review Board of Seoul National University Bundang Hospital (IRB No. B1710/426-101). Owing to the retrospective design, the requirement for informed consent was waived.

\section{Selection of Patients}

We retrospectively reviewed all cases of naïve septic shoulder arthritis treated at our institution between November 2003 and February 2016. Children and patients with isolated acromioclavicular joint infection, tuberculosis infection, or a previous history of septic arthritis were excluded. Thirty-six cases, contributed by 35 patients, were identified. Among them, one male patient had a bilateral presentation without other joint involvement, and one patient had been treated for infective spondylitis. All arthroscopic surgeries were performed by a single surgeon $(\mathrm{JHO})$.

\section{Diagnostic Assessment}

Plain radiographs and magnetic resonance images (MRIs) were obtained, as well as blood analyses, including white blood cell (WBC) with differential counts, the erythrocyte sedimentation rate (ESR), and the C-reactive protein (CRP) level. Assessment of preoperative joint fluid aspirate was feasible in 24 patients. Blood and intraoperative specimen cultures were examined to differentiate the type of infectious organism: aerobic, anaerobic, fungal, or mycobacterial. A provisional septic arthritis diagnosis was made based on clinical symptoms, blood test results, joint fluid aspirate analysis, and MRI findings. All patients underwent arthroscopic debridement and irrigation as the first treatment modality. The diagnosis of septic arthritis was confirmed through intraoperative findings and culture results along with the preoperative radiologic and laboratory results. All cases were classified according to the Gächter staging system, based on intraoperative findings $[9,11]$.

\section{Surgical Technique}

All arthroscopic surgeries were performed in the lateral decubitus position. We attempted to obtain fresh specimens for culturing (pus, fluid, or infected granulation tissue) after trocar insertion to prevent dilution with the irrigation fluid. The posterior portal was used as a viewing portal, and the anterior portal as a working portal, to perform thorough synovectomy and massive irrigation. According to the preoperative MRI findings, we tried to evaluate the subacromial (SA) space and glenohumeral (GH) joint space separately to identify the focus of the infection and the presence of a rotator cuff tear. If only a GH joint space infection was suspected on the preoperative MRI, we first tried to inspect the SA space. After arthroscopic synovectomy for one space infection, the other space was evaluated to confirm that the infection was in only one space. Debridement and irrigation were used in the other space to prevent the spread of infection.

In the $\mathrm{GH}$ joint, a posterolateral portal was created to approach the posteroinferior $\mathrm{GH}$ joint and bare area of the humerus. In the SA space, a conventional lateral portal was created to remove infected granulation tissue. To access the subcoracoid and posterior SA spaces, a $70^{\circ}$ arthroscope was used for better visualization (Fig. 1). Sufficient irrigation fluid (mean volume of fluid, $25.1 \pm 8.1 \mathrm{~L}$; mean operation time, $103.5 \pm 21.4$ minutes) was used for meticulous debridement. In four cases with suspected osteomyelitis based on preoperative MRIs, either a motorized burr was used to debride the infected bony tissues or drilling was performed until uninfected bony tissue was exposed. After extensive irrigation and debridement, two to four 3.2-mm suction drains (average, 3.3 drains) were inserted, separately, into the anterior and posterior parts of the $\mathrm{GH}$ joint and SA spaces, in regions where the infection focus was iden- 

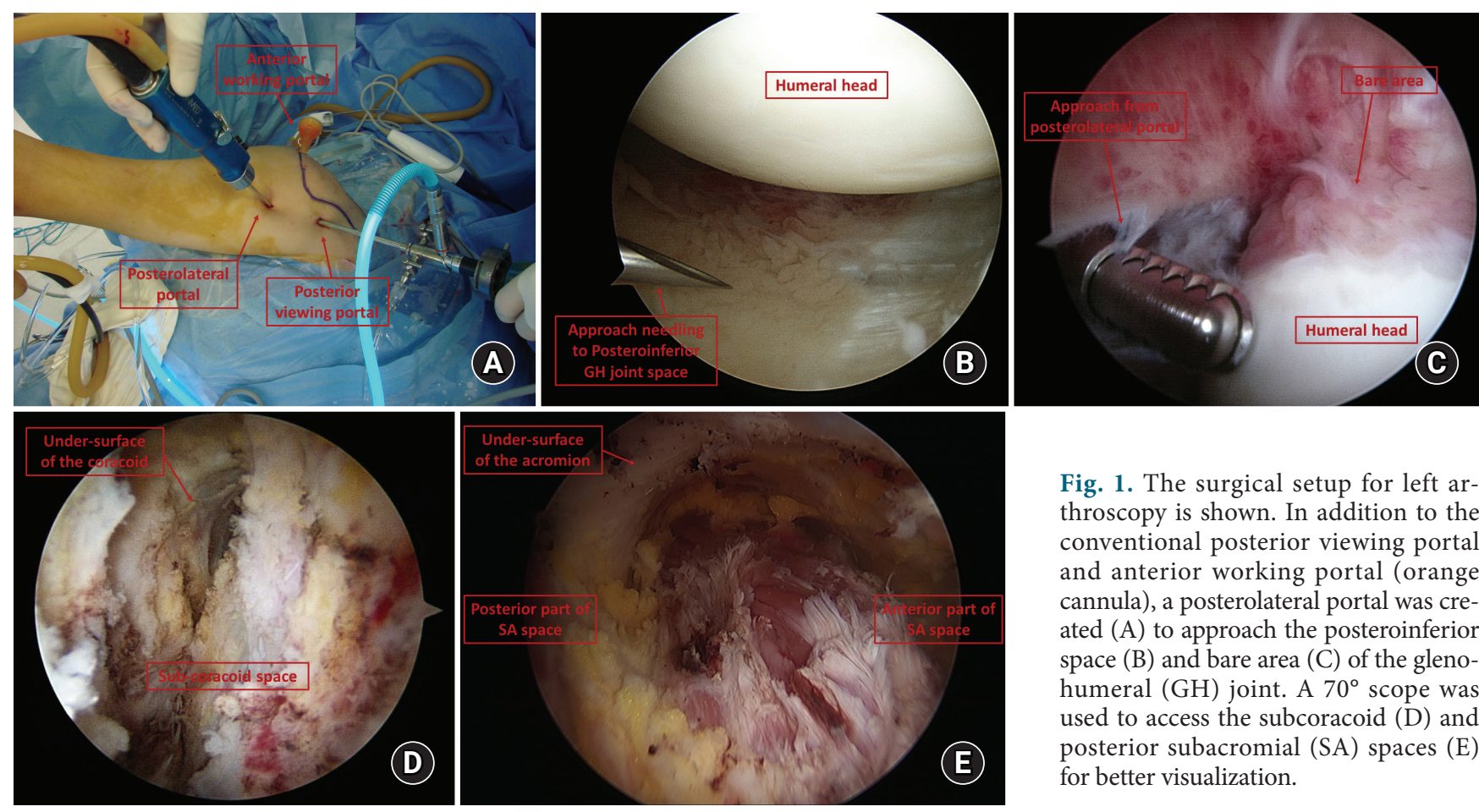

Fig. 1. The surgical setup for left arthroscopy is shown. In addition to the conventional posterior viewing portal and anterior working portal (orange cannula), a posterolateral portal was created (A) to approach the posteroinferior space (B) and bare area (C) of the glenohumeral (GH) joint. A $70^{\circ}$ scope was used to access the subcoracoid (D) and posterior subacromial (SA) spaces (E) for better visualization.

tified during arthroscopy, for continuous postoperative drainage (Fig. 2).

\section{Postoperative Management}

There was no information on patient antibiotic use prior to being transferred to our institution. No antibiotics were administered before surgery; broad-spectrum antibiotics were started immediately after collecting the intraoperative specimens and were subsequently changed according to the culture and sensitivity study results, after consulting with an infectious disease specialist. Intravenous antibiotics were continued until the ESR and CRP levels were normalized $[9,13,14]$. Then, oral antibiotics were determined by the infectious disease specialist. The drains inserted during the arthroscopic procedure were removed sequentially when the daily drainage output was $<5 \mathrm{~mL}$. Passive range of motion (ROM) exercises were initiated after removal of all drains, under the supervision of a physiatrist.

The clinical outcomes, including the ROM and simple shoulder test scores, were evaluated at the final follow-up visit. Three components of the ROM were measured using a goniometer: forward flexion, external rotation at side, and internal rotation at back. Forward flexion was measured as the angle between the forearm and the thorax, with the elbow in full extension. External rotation at side was measured as the angle between the thorax and the forearm, with the arm at the side of the body with $90^{\circ}$ of elbow flexion.

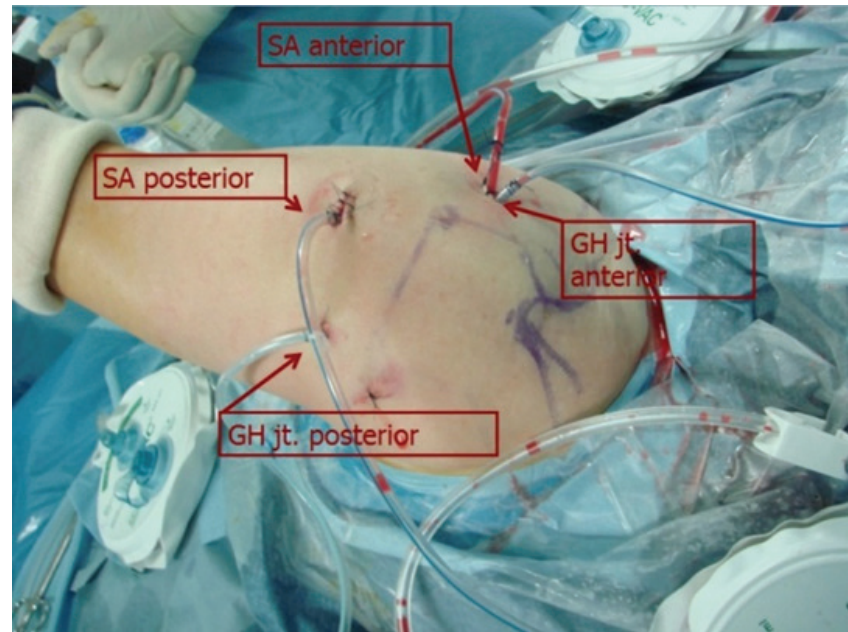

Fig. 2. After extensive debridement and irrigation, four separate suction drains were inserted into the anterior and posterior aspects of the glenohumeral $(\mathrm{GH})$ joint (jt) space and subacromial (SA) space for continuous drainage. Images are of the left shoulder with the patient in a lateral decubitus position.

Internal rotation at back was measured by the vertebral level reached by the thumb of the hand reaching behind the back. The inferior pole of the scapula was referenced as the seventh thoracic vertebra and the iliac crest as the fourth lumbar vertebra $[15,16]$. 


\section{RESULTS}

The 35 patients enrolled in our study group included 14 men (40\%) and had a mean age of $63.8 \pm 13.0$ years (range, $41-91$ years). The mean follow-up period was $14.3 \pm 5.1$ months (range, $12-33$ months). Injection or acupuncture to the involved shoulder was the suspected cause of infection in 19 of the 36 shoulders (52.8\%). Arthroscopic surgery was performed 10.9 \pm 9.6 days (range, 1-35 days) after symptom onset. Eleven of the 35 patients (31.4\%) were immunocompromised, with seven (20\%) having diabetes mellitus (DM), four (11.4\%) having a malignancy (lung cancer, multiple myeloma, adrenal cancer with spleen metastasis, and breast cancer with thyroid cancer), and two patients having liver cirrhosis, one with DM and one with DM and a malignancy.

The preoperative WBC count, ESR, and CRP were $9.39 \pm 4.14$ $\times 10^{9} / \mathrm{L}$ (normal, $4.0-10.0 \times 10^{9} / \mathrm{L}$ ), $60.30 \pm 30.55 \mathrm{~mm} / \mathrm{hr}$ (normal, 0-9 mm/hr), and $9.23 \pm 8.07 \mathrm{mg} / \mathrm{dL}$ (normal, 0-0.5 mg/dL), respectively. The WBC count of the preoperatively aspirated joint fluid was $128.867 \pm 106.09 \times 10^{9} / \mathrm{L}$, with a mean differential neutrophil count of $88.3 \%$. To avoid unnecessary contamination, the extent of the infection was determined based on preoperative MRIs. Both the GH joint and the SA space were involved in most cases (21 cases), while only the GH joint was involved in 14 cases, and only the SA space was involved in one case. A full-thickness rotator cuff tear was present in 15 shoulders (41.7\%). With regard to infection severity, the distribution of Gächter stages was as follows: stage 1, nine shoulders (25\%); stage II, 11 shoulders (30.6\%); stage III, 12 shoulders (33.3\%); and stage IV, four shoulders (11.1\%) (Table 1).

The causative organism was identified in 15 cases $(41.7 \%)$ from either the preoperative aspiration or the intraoperative specimen culture. The most common pathogen identified was Staphylococcus aureus (10 shoulders [27.8\%]), specifically methicillin-sensitive $S$. aureus (MSSA, six shoulders) and methicillin-resistant $S$. aureus (MRSA, four shoulders). Other identified organisms are listed in Table 2. On blood culture analyses, five cases showed positive results (two cases of MRSA infection and

Table 1. Classification using the Gächter staging system [9]

\begin{tabular}{lcc}
\hline Stage & \multicolumn{1}{c}{ Description } & No. (\%) \\
\hline I & $\begin{array}{c}\text { Opacity of fluid, redness of the synovial } \\
\text { membrane, possible petechiae }\end{array}$ & $9(25)$ \\
II & $\begin{array}{c}\text { Purulent material, severe inflammation, } \\
\text { and fibrinous deposition }\end{array}$ & $11(30.6)$ \\
III & $\begin{array}{c}\text { Thickening of the synovial membrane, } \\
\text { with cartilage erosion }\end{array}$ & $12(33.3)$ \\
IV & $\begin{array}{l}\text { Most aggressive stage, with subchondral } \\
\text { delamination }\end{array}$ & $4(11.1)$ \\
\hline
\end{tabular}

one case each of MSSA, Streptococcus pneumoniae, and Streptococcus dysgalactiae infection).

CRP levels normalized at $3.7 \pm 2.9$ weeks after surgery, and intravenous antibiotics were used for $3.9 \pm 1.8$ weeks, until the ESR and CRP levels normalized, with further use of oral antibiotics for an additional $3.6 \pm 1.9$ weeks. Drains were removed sequentially according to the daily output, with all drains removed by $8.9 \pm 4.3$ days after surgery. The average length of hospital stay, which depended on the duration of intravenous antibiotic treatment recommended by the infectious disease specialist, was $4.0 \pm 2.6$ weeks. At the final follow-up, the mean ROM was $150.0^{\circ} \pm 37.3^{\circ}$ for forward flexion, $65.3^{\circ} \pm 16.1^{\circ}$ for external rotation, and $\mathrm{T} 9 \pm 2$ for internal rotation. The mean simple shoulder test score was $7.9 \pm 3.6$ points.

Among the 36 shoulders treated, reoperation was required in two cases (5.6\%), both with Gächter stage III infection. Reoperation was performed when the drain output and CRP level did not decrease, and there was evidence of persisting infection on postoperative MRIs. One of these two cases presented with progressive osteomyelitis despite an intact rotator cuff and localized infection to the $\mathrm{GH}$ joint space. This patient underwent reoperation 2 weeks after the first arthroscopic procedure, using an open arthrotomy and massive curettage of the bone lesion on the humeral head. The second patient suffered from infective spondylitis. After an initial successful arthroscopic debridement, he had taken intravenous antibiotics for 4 weeks, followed by oral antibiotics for 3 weeks under the supervision of the infectious disease specialist. Seven weeks after surgery, he had elevated ESR and CRP levels, with aggravated shoulder pain after his CRP level normalized. This patient underwent revision arthroscopic debridement. The infection in each of these two cases was eradicated successfully after the second surgery.

\section{DISCUSSION}

Thirty-six cases of septic arthritis of the shoulder were successfully

Table 2. Causative organisms identified by preoperative aspiration or intraoperative specimen culture

\begin{tabular}{lc}
\hline Organism & No. (\%) \\
\hline Staphylococcus aureus & $10(27.8)$ \\
Methicillin-sensitive S. aureus & $6(16.7)$ \\
Methicillin-resistant S. aureus & $4(11.1)$ \\
Staphylococcus epidermidis & $1(2.8)$ \\
Coagulase-negative Staphylococcus & $1(2.8)$ \\
Streptococcus pneumoniae & $1(2.8)$ \\
Serratia marcescens & $1(2.8)$ \\
Streptococcus dysgalactiae & $1(2.8)$ \\
No growth & $21(58.3)$ \\
\hline
\end{tabular}


treated using the arthroscopic debridement approach with an additional posterolateral portal, use of a $70^{\circ}$ arthroscope, irrigation with $>20 \mathrm{~L}$ of normal saline, placement of multiple separate suction drains, and use of appropriate antibiotics. Only two cases required reoperation (5.5\%), a rate which was strikingly lower than rates of $26 \%$ to $32 \%$ that have been previously reported $[3,11]$ for arthroscopic treatment of septic arthritis of the shoulder.

The surgical methods for septic arthritis of shoulder include both open arthrotomy and arthroscopic debridement. There is no consensus on which method is the best treatment for septic shoulder. Several studies have been conducted regarding surgical methods. Böhler et al. [17] compared 38 cases of arthrotomy to 21 cases of arthroscopic debridement. They showed that open arthrotomy is the more effective surgical method. However, Bovonratwet et al. [18] reported similar rates of reoperation and postoperative complications between the two surgical methods. Jiang et al. [19] also reported no difference in the reoperation rate between these two surgical methods. Some studies showed that arthroscopic surgery is ineffective on higher Gächter stages of septic arthritis [9,20]. However, Jeon et al. [3] conducted arthroscopic debridement in patients with higher Gächter stages (nine cases in stage III and two cases in stage IV). They reported reoperation in two of nine cases in stage III and in one of two cases in stage IV. In our study, all patients underwent primary arthroscopic surgery. There were 12 cases (33.3\%) in Gächter stage III and four cases (11.1\%) in Gächter stage IV. Two cases of Gächter stage III required reoperation. We believe that arthroscopic surgery would be an excellent treatment for septic shoulder arthritis even in higher Gächter stages if sufficient irrigation and debridement are performed and proper drains are used.

When we included data from studies $[4,7,21]$ in which a mixed treatment approach was used, including open arthrotomy, our rate of reoperation of $5.6 \%$ was still low compared to reported rates ranging from $14.7 \%$ to $32 \%$. We reviewed several studies that reported the reoperation rate for their case series (Table 3). Jeon et al. [3] reported a $26 \%$ rate of reoperation among 19 cases where arthroscopic treatment was performed for septic shoulder arthritis, with the number of arthroscopic procedures required to achieve infection resolution being correlated to the stage of infection. Abdel et al. [11] reported that, among 50 patients, nearly one in three required additional surgical intervention. In the study by Klinger et al. [4], 12 cases of septic shoulder were treated with the arthroscopic technique, and the other 11 cases used a combination of arthroscopic and open techniques. The need for an additional open technique was determined based on the clinical extent of the infection, duration of symptoms, the intraoperative Gächter stage, and the observation of an abscess or spread of the septic area on preoperative MRI. The authors noted a $26 \%$ rate of reoperation overall and a $25 \%$ rate of reoperation among cases treated with only the arthroscopic technique. Cho and Oh [7] reported a $14.7 \%$ rate of reoperation among 34 septic shoulders, with 22 treated by arthroscopy and 12 by an open approach; an 18.2\% rate of reoperation was noted among cases treated with arthroscopy only. The open method was performed when there was evidence of osteomyelitis or abscess formation in the subcoracoid space on preoperative MRI. Duncan and Sperling [21] reported a 21\% rate of reoperation among 19 septic shoulders treated with an open (nine cases) or arthroscopic (10 cases) technique, with the approach selected based on the surgeon's preference.

Currently, there is no standardized treatment method or protocol for septic arthritis of the shoulder. They tend to be selected based on the surgeon's preference and experience. Based on our data, we emphasize the importance of complete debridement and sufficient irrigation, with drainage, when treating septic shoulders. Complete debridement using an additional posterolateral portal, $70^{\circ}$ arthroscope, abundant irrigation (with $>20 \mathrm{~L}$ of normal sa-

Table 3. Comparison to previous studies on septic arthritis of the shoulder joint

\begin{tabular}{|c|c|c|c|c|c|c|c|c|c|c|}
\hline \multirow[b]{2}{*}{ Study } & \multirow{2}{*}{$\begin{array}{l}\text { No. of } \\
\text { cases }\end{array}$} & \multirow{2}{*}{$\begin{array}{l}\text { Method } \\
\text { (open:ar- } \\
\text { throscopic) }\end{array}$} & \multirow{2}{*}{$\begin{array}{l}\text { Reoperation } \\
\text { rate (only in ar- } \\
\text { throscopic, \%) }\end{array}$} & \multirow{2}{*}{$\begin{array}{c}\text { Mean age } \\
(\mathrm{yr})\end{array}$} & \multirow{2}{*}{$\begin{array}{l}\text { Mean } \\
\text { follow-up } \\
(\mathrm{mo})\end{array}$} & \multirow{2}{*}{$\begin{array}{l}\text { Mean symp- } \\
\text { tom duration } \\
\text { (day) }\end{array}$} & \multirow{2}{*}{$\begin{array}{l}\text { Irrigation } \\
\text { (L) }\end{array}$} & \multicolumn{2}{|c|}{ Suction drain } & \multirow{2}{*}{$\begin{array}{l}\text { Culture } \\
\text { positive } \\
\text { rate (\%) }\end{array}$} \\
\hline & & & & & & & & Number & $\begin{array}{c}\text { Duration } \\
\text { (day) }\end{array}$ & \\
\hline $\begin{array}{l}\text { Duncan and } \\
\text { Sperling [21] }\end{array}$ & 19 & $\operatorname{Mix}(9: 10)$ & 21 & 75.5 (49-94) & 6 & NA & NA & NA & NA & 100 \\
\hline Klinger et al. [4] & 23 & $\operatorname{Mix}(12: 11)$ & $26(25)$ & $64(41-85)$ & 3 & $16(5-76)$ & 10 & NA & 2 & 87 \\
\hline Cho and Oh [7] & 24 & $\operatorname{Mix}(22: 12)$ & $14.7(18.2)$ & $61.8(32-79)$ & 32.4 & $23.3(1-120)$ & 12 & NA & $15.6 \pm 9.7$ & 38 \\
\hline Jeon et al. [3] & 19 & Arthroscopic & 26 & $59(23-85)$ & 16.4 & $21(7-56)$ & $5-20$ & NA & NA & 68 \\
\hline Abdel et al. [11] & 50 & Arthroscopic & 32 & $66(25-97)$ & 31 & $8(1-60)$ & 10 & Only 1 & NA & 100 \\
\hline This study & 36 & Arthroscopic & 5.5 & $63.8(41-91)$ & 11.4 & $10.9(1-35)$ & $25.1 \pm 8.1$ & $\begin{array}{c}3.3 \\
\text { (Maximum 4) }\end{array}$ & $8.9 \pm 4.3$ & 42 \\
\hline
\end{tabular}

Values are presented as median (range) or mean \pm standard deviation unless otherwise indicated.

NA, not applicable. 
line), and sufficient drainage (using multiple separate suction drains) may reduce the rate of reoperation.

Using a $70^{\circ}$ arthroscope and a posterolateral portal is useful to achieve complete debridement. To access the posteroinferior SA space, where the infraspinatus and teres minor exist, open debridement was preferred. Cho and Oh [7] chose an open debridement in patients with subcoracoid abscesses to achieve thorough debridement and irrigation [6]. However, a 70 arthroscope can easily access the subcoracoid and posteroinferior SA space. Furthermore, we created an additional posterolateral portal to approach the posterior and inferior GH joint space and bare area of the humerus, which allowed us to complete a meticulous debridement of this difficult-to-reach area. We performed thorough debridement and irrigation without conversion to the open arthrotomy.

Among 36 patients, only one space was involved in 41.7\% (GH joint only in 14 cases and the SA space only in one case). In patients with intact rotator cuff tear, these two spaces may be fully separated. However, contamination into the other space is difficult to avoid. Even if the two spaces are separated, arthroscopic instruments should be passed into the SA space to access the GH joint. Because irrigation with normal saline is performed under positive pressure, the surgeon should be careful to prevent the spread of infection.

Sufficient irrigation with drainage also contributes to successful surgical management of septic arthritis. Previous studies [3,4,7,11] have used up to $10 \mathrm{~L}$ of normal saline for irrigation, whereas we used $25.1 \pm 8.1 \mathrm{~L}$ of saline for irrigation to ensure a thorough debridement. Recently, Joo et al. [22] reported that a large volume of irrigation (>16.8 L) was important to lower recurrence after arthroscopic surgery of septic shoulder. Utilizing large volumes of irrigation solution is better for infection control, despite being time consuming. Furthermore, we inserted multiple separate 3.2-mm-diameter suction drains (average, 3.3 drains) after the procedure, usually two drains into the anterior and posterior GH joint spaces, and two drains into the anterior and posterior SA space, wherever the infection focus was identified during arthroscopy. These drains were removed sequentially, according to the daily output. In most previous studies, information regarding the number, location, and duration of indwelling drains was not reported (Table 3). Jung et al. [23] reported on the successful treatment and management of septic arthritis of the shoulder using continuous negative pressure drainage. They inserted a small-diameter $(3.2 \mathrm{~mm})$ drain into the $\mathrm{GH}$ joint space and a large-diameter $(6.7 \mathrm{~mm}, 20$-Fr chest tube) drain into the SA space, with $15-\mathrm{cm} \mathrm{H}_{2} \mathrm{O}$ of continuous negative pressure applied. Similarly, in our arthroscopic protocol, multiple drains placed in separate locations and connected to separate suction bags facilitated proper drainage after surgery and, consequent- ly, lowered the rate of reoperation, with smaller size drains used and a shorter indwelling duration of the drain.

The use of appropriate antibiotics for the treatment of septic arthritis is important. The overall recommended duration for antibiotics is at least 4-6 weeks, and oral antibiotics may be considered if symptoms improve after intravenous antibiotics have been administered for at least 2 weeks [24]. In the current study, the antibiotics were chosen according to the results of microbial culture with consultation from an infectious disease specialist. We changed intravenous antibiotics to oral antibiotics after normalization of the CRP level, as advised by the infectious disease specialist. CRP is the most widely used parameter to evaluate treatment of septic arthritis $[24,25]$. We used intravenous antibiotics for $3.9 \pm 1.8$ weeks, followed by oral antibiotics for $3.6 \pm 1.9$ weeks. In the current study, mixing antibiotics into the irrigation fluid was not performed because sufficient antibiotics level in the synovial fluid can be reached after intravenous administration [26,27]. Moreover, a chemical synovitis may occur after intra-articular use of antibiotics [28].

In our study, the most common causative organism of septic arthritis was S. aureus (10 shoulders), specifically MSSA (six shoulders) and MRSA (four shoulders), and this overall trend was similar to that of previous studies $[3,4,6,7,11,21,23]$. However, the rate of positive results for the culture was relatively low (42\%). As our institution is a tertiary hospital, many patients were referred from another hospital and had been prescribed antibiotics prior to aspiration or operation, which may explain the low positive culture rate [7]. Cutibacterium acnes may also be the cause for the low positive results rate because we could not perform long-term cultures of specimens. C. acnes is an anaerobic bacterium found in moist skin areas, including the axilla, sebaceous gland, and hair follicles. It is one of the most common shoulder infection pathogens identified after arthroscopic operation [29,30] and is occasionally found in naive septic arthritis of the shoulder [21]. Importantly, C. acnes is a slow-growing organism and, thus, longer culture duration is needed. Therefore, multiple culture specimens must be kept for over 2 weeks to determine the causative organism to inform the selection of effective antibiotics.

\section{Limitations}

The major limitation of our study was the absence of a control group, which was not possible for ethical reasons. In addition, our reoperation rate was so low that we were unable to include a comparison to eradiated patients and recurred patients. We instead compared our results to those from previous studies to emphasize that our techniques may lead to better outcomes relative to those of previous studies. The lack of long-term follow-up was another limitation. However, infection control of septic arthritis is usually 
completed within 6 months following surgery, and any required reoperation is usually performed within this time. Furthermore, assessments of the definite treatment outcome for the accompanying rotator cuff tear or osteoarthritis were not included in this study. Other limitations included the retrospective study design, small sample size, limited ability to compare preoperative and postoperative clinical information, and lack of specific endpoints for the outcome or eradication of infection markers. However, as septic arthritis of the shoulder joint is a relatively rare disease requiring urgent treatment, these limitations were inevitable and do not alter the importance of our results.

Our findings indicate that to reduce the reoperation rate of septic arthritis of the shoulder, complete debridement and sufficient irrigation with proper drainage are essential. We performed complete debridement with thorough GH synovectomy, using an additional posterolateral portal and SA bursectomy with a $70^{\circ}$ arthroscope, as well as sufficient irrigation with $>20 \mathrm{~L}$ of normal saline and proper drainage using multiple separate suction drains in each location. Arthroscopic treatment for septic arthritis of the shoulder may yield better outcomes, especially in terms of the rate of reoperation.

\section{REFERENCES}

1. Lossos IS, Yossepowitch O, Kandel L, Yardeni D, Arber N. Septic arthritis of the glenohumeral joint: a report of 11 cases and review of the literature. Medicine (Baltimore) 1998;77:177-87.

2. Cleeman E, Auerbach JD, Klingenstein GG, Flatow EL. Septic arthritis of the glenohumeral joint: a review of 23 cases. J Surg Orthop Adv 2005;14:102-7.

3. Jeon IH, Choi CH, Seo JS, Seo KJ, Ko SH, Park JY. Arthroscopic management of septic arthritis of the shoulder joint. J Bone Joint Surg Am 2006;88:1802-6.

4. Klinger HM, Baums MH, Freche S, Nusselt T, Spahn G, Steckel H. Septic arthritis of the shoulder joint: an analysis of management and outcome. Acta Orthop Belg 2010;76:598-603.

5. Leslie BM, Harris JM 3rd, Driscoll D. Septic arthritis of the shoulder in adults. J Bone Joint Surg Am 1989;71:1516-22.

6. Rhee YG, Cho NS, Kim BH, Ha JH. Injection-induced pyogenic arthritis of the shoulder joint. J Shoulder Elbow Surg 2008;17: 63-7.

7. Cho $\mathrm{CH}$, Oh GM. Prognostic factors affecting the clinical outcome of septic arthritis of the shoulder. J Hand Surg Asian Pac Vol 2016;21:339-44.

8. Bremell T, Abdelnour A, Tarkowski A. Histopathological and serological progression of experimental Staphylococcus aureus arthritis. Infect Immun 1992;60:2976-85.
9. Stutz G, Kuster MS, Kleinstück F, Gächter A. Arthroscopic management of septic arthritis: stages of infection and results. Knee Surg Sports Traumatol Arthrosc 2000;8:270-4.

10. Vispo Seara JL, Barthel T, Schmitz H, Eulert J. Arthroscopic treatment of septic joints: prognostic factors. Arch Orthop Trauma Surg 2002;122:204-11.

11. Abdel MP, Perry KI, Morrey ME, Steinmann SP, Sperling JW, Cass JR. Arthroscopic management of native shoulder septic arthritis. J Shoulder Elbow Surg 2013;22:418-21.

12. Hunter JG, Gross JM, Dahl JD, Amsdell SL, Gorczyca JT. Risk factors for failure of a single surgical debridement in adults with acute septic arthritis. J Bone Joint Surg Am 2015;97:558-64.

13. Jeon YD, Moon JY, Son JH, Kim JM, Choi Y. The efficacy of arthroscopic debridement with continuous irrigation in failed arthroscopic debridement for septic arthritis of the knee. J Korean Orthop Assoc 2016;51:308-14.

14. Chou AC, Mahadev A. The use of C-reactive protein as a guide for transitioning to oral antibiotics in pediatric osteoarticular infections. J Pediatr Orthop 2016;36:173-7.

15. Godfrey J, Hamman R, Lowenstein S, Briggs K, Kocher M. Reliability, validity, and responsiveness of the simple shoulder test: psychometric properties by age and injury type. J Shoulder Elbow Surg 2007;16:260-7.

16. Park JS, Park HJ, Kim SH, Oh JH. Prognostic factors affecting rotator cuff healing after arthroscopic repair in small to medium-sized tears. Am J Sports Med 2015;43:2386-92.

17. Böhler C, Pock A, Waldstein W, et al. Surgical treatment of shoulder infections: a comparison between arthroscopy and arthrotomy. J Shoulder Elbow Surg 2017;26:1915-21.

18. Bovonratwet P, Fu MC, Pathak N, et al. Surgical treatment of septic shoulders: a comparison between arthrotomy and arthroscopy. Arthroscopy 2019;35:1984-91.

19. Jiang JJ, Piponov HI, Mass DP, Angeles JG, Shi LL. Septic arthritis of the shoulder: a comparison of treatment methods. J Am Acad Orthop Surg 2017;25:e175-84.

20. Gaechter A. Arthroscopic lavage for joint infections. Orthop Traumatol 1993;2:104-6.

21. Duncan SF, Sperling JW. Treatment of primary isolated shoulder sepsis in the adult patient. Clin Orthop Relat Res 2008;466:13926.

22. Joo YB, Lee WY, Shin HD, Kim KC, Kim YK. Risk factors for failure of eradicating infection in a single arthroscopic surgical procedure for septic arthritis of the adult native shoulder with a focus on the volume of irrigation. J Shoulder Elbow Surg 2020;29:497-501.

23. Jung HJ, Song JH, Kekatpure AL, et al. The use of continuous negative pressure after open debridement for septic arthritis of 
the shoulder. Bone Joint J 2016;98:660-5.

24. Korean Society for Chemotherapy; Korean Society of Infectious Diseases; Korean Orthopaedic Association. Clinical guidelines for the antimicrobial treatment of bone and joint infections in Korea. Infect Chemother 2014;46:125-38.

25. Söderquist B, Jones I, Fredlund H, Vikerfors T. Bacterial or crystal-associated arthritis? Discriminating ability of serum inflammatory markers. Scand J Infect Dis 1998;30:591-6.

26. Nelson JD. Antibiotic concentrations in septic joint effusions. N Engl J Med 1971;284:349-53.
27. Patel H, Nade S. Acute staphylococcal septic arthritis: the effect of cloxacillin therapy in an avian model. J Orthop Res 1988;6:6372.

28. Argen RJ, Wilson CH Jr, Wood P. Suppurative arthritis: clinicalfeatures of 42 cases. Arch Intern Med 1966;117:661-6.

29. Athwal GS, Sperling JW, Rispoli DM, Cofield RH. Deep infection after rotator cuff repair. J Shoulder Elbow Surg 2007;16:306-11.

30. Kwon YW, Kalainov DM, Rose HA, Bisson LJ, Weiland AJ. Management of early deep infection after rotator cuff repair surgery. J Shoulder Elbow Surg 2005;14:1-5. 